\begin{tabular}{ll} 
C O L L O Q U I U M & M A T H E M A T I C U M \\
\hline Vol. LXVII & 1994 \\
\hline
\end{tabular}

\title{
EXISTENCE AND NONEXISTENCE OF SOLUTIONS FOR A MODEL OF GRAVITATIONAL INTERACTION OF PARTICLES, II
}

BY

\author{
PIOTR BILER (WROCEAW), DANIELLE HILHORST (ORSAY) \\ AND TADEUSZ NADZIEJA (WROCŁAW)
}

We study the existence and nonexistence in the large of radial solutions to a parabolic-elliptic system with natural (no-flux) boundary conditions describing the gravitational interaction of particles. The blow-up of solutions defined in the $n$-dimensional ball with large initial data is connected with the nonexistence of radial stationary solutions with a large mass.

1. Introduction. This is the second part of [5]. We study here radial solutions of the parabolic-elliptic system from [5, (1)-(5)], their existence and nonexistence for both stationary and evolution cases. The system, defined in a bounded domain $\Omega$ of $\mathbb{R}^{n}$, is

$$
\begin{gathered}
u_{t}=\Delta u+\nabla \cdot(u \nabla \varphi), \\
\Delta \varphi=u,
\end{gathered}
$$

with the nonlinear no-flux condition

$$
\frac{\partial u}{\partial \nu}+u \frac{\partial \varphi}{\partial \nu}=0
$$

where $\nu$ denotes the outward unit normal vector to $\partial \Omega$. For the potential $\varphi$ we assume either

$$
\varphi=0 \quad \text { on } \partial \Omega,
$$

or instead of the Dirichlet boundary condition above,

$$
\varphi=E_{n} * u
$$

with $E_{n}$ being the fundamental solution of the $n$-dimensional Laplacian. For the initial-boundary value problem we add the condition

$$
u(x, 0)=u_{0}(x) \geq 0 .
$$

1991 Mathematics Subject Classification: 35B40, 35K60, 35Q99, 82C21.

Key words and phrases: parabolic-elliptic system, nonlinear boundary conditions, global existence of solutions, blowing-up solutions.

Research of the first and third authors was partially supported by KBN grants. 
For the interpretation of (1)-(5) we refer the reader to the introduction of $[5]$.

When $\Omega$ is a radially symmetric domain: a ball, an annulus, or a spherical shell in $\mathbb{R}^{n}$, we consider radially symmetric solutions. We define the integrated density $Q(r, t)=\int_{B_{r}} u d x$ which satisfies the following parabolic equation with the Dirichlet boundary conditions on $[0, R] \times[0, T]$ if $\Omega=B_{R}$ :

$$
\begin{aligned}
& Q_{t}=Q_{r r}-(n-1) r^{-1} Q_{r}+\sigma_{n}^{-1} r^{1-n} Q Q_{r}, \\
& Q(0, t)=0, \quad Q(R, t)=Q_{0}, \\
& Q(r, 0)=Q_{0}(r) \geq 0, \quad Q_{0}(r) \text { nondecreasing. }
\end{aligned}
$$

Such a formulation permits us to deal with less regular densities $u$ (including measures, not only $L^{1}$ functions) than those considered in [5].

To justify this approach observe that the system (1)-(4) is rotation invariant. Hence, for a radially symmetric initial condition (5) the unique solution constructed by any method described in [5, Theorem 2(i), (ii)] $(p>n)$ is radial. After integrating this solution we obtain a (more regular) new dependent variable $Q(r, t)=\int_{B_{r}} u d x=\sigma_{n} \int_{0}^{r} \varrho^{n-1} u(\varrho, t) d \varrho$, which is equal to the mass contained in the ball of current radius $r \in[0, R]$. It is easy to verify that $Q$ satisfies (6) (observe that $\sigma_{n} r^{n-1} \phi_{r}=Q$ if $\phi(r, t)=\varphi(x, t)$ with $|x|=r)$, together with the physically obvious compatibility conditions

$$
M=\int_{B_{R}} u_{0} d x, \quad Q_{0}(R)=Q_{0}=M
$$

All this shows that (6) with the above conditions (7) does have a unique solution in the class of solutions described in [5, Theorem 2]. The total mass $M$ is considered as a meaningful parameter in the problem (6)-(7).

2. Radially symmetric stationary solutions. We recall some previous results on the radially symmetric stationary solutions in the two- and three-dimensional cases from [11], [13], where the problem

$$
\begin{aligned}
& \left(r^{n-1} \Phi^{\prime}(r)\right)^{\prime} \int_{0}^{R} \varrho^{n-1} \exp (-\Phi(\varrho)) d \varrho \\
& \quad=M \sigma_{n}^{-1} r^{n-1} \exp (-\Phi(r)), \quad '=d / d r \\
& \Phi^{\prime}(0)=0, \quad \Phi(R)=0 \quad
\end{aligned}
$$

has been studied. This problem can be deduced from the stationary equations corresponding to (1)-(4) (cf. [5, (10), (11)]).

In the two-dimensional case an explicit form of solution to (8) is available, since the equation (8) is integrable for $n=2$. This implies that (8) has a unique solution for all $0<M<8 \pi$ and it has no solution for $M \geq 8 \pi$. 
In the three-dimensional case the situation is much more complicated. Namely, for sufficiently small $M$ the problem (8) has a unique solution, and there exists $M_{0}>8 \pi$ such that for $M>M_{0}$ there are no solutions. For some intermediate values of $M$ from the interval $\left(0, M_{0}\right)$ there are several solutions, and for $M=8 \pi$ there exist infinitely many solutions.

A general nonexistence result in star-shaped domains from [5, Section 2] gives nonexistence of solutions in the ball $B_{R} \subset \mathbb{R}^{n}$ when $M \geq 2 n \sigma_{n} R^{n-2}$.

Applying the contraction principle, it has been proved in [13] that if $\Omega$ is a spherical shell, i.e. $\Omega=\left\{x \in \mathbb{R}^{n}: 0<a<|x|<R\right\}$, then (8) with a suitable condition at $r=a$ has a unique radially symmetric solution for all positive $M$ 's. The existence of solutions for all $M>0$ is proved, using different methods, also in [6] and [11]. It is an interesting fact that in the case of shells, nonradially symmetric solutions can exist (see [15]-[18] for a related problem in general domains).

Using phase plane methods developed in [11], it is possible to prove that in every dimension the problem (8) has a unique solution for sufficiently small $M>0$. Moreover, for $n \geq 10$ there exists at most one solution for arbitrary $M>0$. Note that [10] dealt with similar equations but without normalization constant $\mu$. The arguments extending those from [10], [11] mentioned above are much simpler in the (stationary) formulation of (6)-(7) (see (9) below), more general than (8) since less regularity of $\Phi$ is required. For $Q(r, t)$ independent of $t$ the problem (6)-(7) becomes

$$
\begin{aligned}
& Q_{r r}-(n-1) r^{-1} Q_{r}+\sigma_{n}^{-1} r^{1-n} Q Q_{r}=0, \\
& Q(0)=0, \quad Q(R)=M .
\end{aligned}
$$

For simplicity of notation we suppose (with no loss of generality) that $R=1$. Indeed, if $Q$ satisfies (9), then $\widetilde{Q}(r)=R^{2-n} Q(R r)$ solves the equation (9) with the boundary conditions $\widetilde{Q}(0)=0, \widetilde{Q}(1)=M R^{2-n}$, this last being rescaled by $R^{2-n}$.

THEOREM 1. Let $R=1$ in the problem (9).

(i) There exists $M_{0}(n)$ such that (9) admits no solution for $M>M_{0}(n)$. In fact, $M_{0}(2)=8 \pi, M_{0}(n)=2 \sigma_{n}$ for $n \geq 10$.

(ii) If $3 \leq n \leq 9$, then solutions of (9) are unique for small $M>0$. For $M=2 \sigma_{n}$ there exist infinitely many solutions; for $M \neq 2 \sigma_{n}$ the number of solutions is finite but grows to infinity as $M$ approaches $2 \sigma_{n}$.

(iii) If $n=2$ or $n \geq 10$, then for every $M>0$ there exists at most one solution of (9).

Proof. For $n=2$ the problem (9) (as well as (8)) is integrable and the explicit form of solution is $Q(r)=8 \pi M\left(8 \pi-M+M r^{2}\right)^{-1} r^{2}$ (cf. also the discussion of singular solutions after the proof of Theorem 3$)$. 
For $n>2$ we will employ the phase plane method (cf. [10], [11]). Introducing the new independent variable $s=\log r$ and putting $v(r)=$ $\sigma_{n}^{-1} r^{3-n} Q_{r}(r)$ and $w(r)=\sigma_{n}^{-1} r^{2-n} Q(r)$ we can verify that $v$ and $w$ satisfy the system

$$
v^{\prime}=2 v-v w, \quad w^{\prime}=(2-n) w+v, \quad{ }^{\prime}=d / d s,
$$

together with the boundary conditions

$$
w(-\infty)=0, \quad w(0)=\sigma_{n}^{-1} M
$$

(the only trajectory such that $w(s) \geq 0$ for $s \rightarrow-\infty$ satisfies $w(-\infty)=0$ ).

The dynamical system (10) has two stationary points $P_{1}=(0,0)$ and $P_{2}=(2(n-2), 2)$. The eigenvalues of the linearization of $(10)$ at these points are $\alpha_{1}=2, \alpha_{2}=2-n$ and $\beta_{1,2}=\frac{1}{2}\left(2-n \pm((n-2)(n-10))^{1 / 2}\right)$. The origin $P_{1}$ is hence a saddle. The solution curve $\gamma(s)=\{(v(s), w(s))$ : $s \in \mathbb{R}, v(s), w(s)>0\}$, starting at the origin and tangent to the eigenvector $(1,1 / n)$ corresponding to the eigenvalue 2 , is a separatrix.

The stationary point $P_{2}$ is a sink and

$L(v, w)=\frac{1}{2}(w-2)^{2}+(v-2(n-2))-2(n-2) \log (v /(2(n-2))), \quad v>0$, with $L\left(P_{2}\right)=0$, is a Lyapunov function for (10). Hence the basin of attraction of $P_{2}$ contains the whole positive quadrant and we conclude that $\gamma$ connects $P_{1}$ with $P_{2}$. For $3 \leq n \leq 9, \operatorname{Im} \beta_{1,2} \neq 0$ and the curve $\gamma$ turns around $P_{2}$ infinitely many times, so we obtain (ii). Note that an analysis of this Lyapunov function $L$ allows us to obtain some estimates for the uniqueness interval $\left(0, M_{0}\right)$ and for the nonexistence range $\left(M_{1}, \infty\right)$, with $M_{1}$ much less than $2 n \sigma_{n}$ given by Pokhozhaev type arguments in [5, Section 2].

For $n \geq 10$ the triangle $\Delta$ bounded by the lines $v=(n-2) w, w=0$ and $2(n-2)(w-2)+\beta_{1}(v-2(n-2))=0$ is positively invariant for the flow generated by $(10)$. To verify this note that $\left(2(2-n), \beta_{1}\right)$ is an eigenvector of the linearization of (10) at $P_{2}$. Hence $\gamma$ connects $P_{1}$ with $P_{2}$ and stays in $\Delta$, so (i) follows. The inequality $w^{\prime}>0$ holds in $\Delta$, so (10)-(11) has a unique solution for $M \leq 2 \sigma_{n}$.

3. Global existence in the radial case. In this section we prove a result on the global existence of solutions in the radial case for not too large data. The construction is based on a modification of the formulation (6)-(7) (with integrated densities) of the problem in the ball and uses the theory of classical Hölder continuous solutions. A related approach for $n=2$ is sketched in [20, Theorem 3.2]. A part of the proof gives also arguments showing existence and regularity of solutions for the problem in an annulus.

Compared to [4, Remark 8] and [5, Theorem 2(iii), (iv)] dealing with the formulation (1)-(5), here less regular densities $u$ can be studied. 
We rewrite (6)-(7) with $R=1$ (which results in no loss of generality, cf. the discussion preceding Theorem 1) using a new independent variable $y=r^{n}$, so that we obtain

$$
\begin{aligned}
& Q_{t}=n^{2} y^{2-2 / n} Q_{y y}+n \sigma_{n}^{-1} Q Q_{y}, \\
& Q(0, t)=0, \quad Q(1, t)=M, \\
& Q(y, 0)=Q_{0}(y) \geq 0, \quad Q_{0}(1)=M .
\end{aligned}
$$

The problem (12) is completely equivalent to $(6)-(7)$ but the equation is slightly simpler than (6).

Let us remark that in the one-dimensional symmetric case, the problem (1)-(5) on $\Omega=(-1,1)$ in the new dependent variable $Q(y, t)=\int_{0}^{y} u(x, t) d x$ for even functions $u$ assumes the form

$$
\begin{aligned}
& Q_{t}=Q_{y y}+Q Q_{y}, \\
& Q(0, t)=0, \quad Q(1, t)=M, \\
& Q(y, 0)=Q_{0}(y) .
\end{aligned}
$$

Its analysis resembles that in [12] for the one-dimensional electrolytic case. Observe that if a function $v$ solves the linear problem for the heat equation

$$
\begin{aligned}
& v_{t}=v_{y y}, \\
& v_{y}(0, t)=0, \quad v_{y}(1, t)-\frac{M}{2} v(1, t)=0, \\
& v(y, 0)=\exp \left(\frac{1}{2} \int_{0}^{y} Q_{0}(x) d x\right),
\end{aligned}
$$

then $Q=2 v_{y} / v$, defined by the Hopf-Cole substitution, is a solution of (13). Separating the variables we see that the solution of (14) can be represented in the form

$$
v(y, t)=a_{0} \exp \left(\lambda^{2} t\right) \cosh \lambda y+\sum_{k=1}^{\infty} a_{k} \exp \left(-\lambda_{k}^{2} t\right) \cos \lambda_{k} y,
$$

where $\lambda$ is a positive (unique) solution of the equation $\lambda \tanh \lambda=M / 2$, $a_{0}>0$, and $\lambda_{k} \tan \lambda_{k}=-M / 2, k=1,2, \ldots, 0<\lambda_{1}<\lambda_{2}<\ldots$

From the explicit form of $v$ we get $\lim _{t \rightarrow \infty} Q(y, t)=2 \lambda \tanh \lambda y$. This means (since $Q_{y}=u$ is the original variable from $\left.(1)\right) \lim _{t \rightarrow \infty} u(y, t)=$ $2 \lambda^{2}(\cosh \lambda y)^{-2}=: \bar{u}(y)$. The function $\Phi=-\log \bar{u}$ satisfies the stationary equation $\frac{d^{2}}{d y^{2}} \Phi=\exp (-\Phi)$ with the conditions $\Phi^{\prime}(0)=0, \Phi(1)=0$, and $\int_{0}^{1} \exp (-\Phi)=M$.

THEOREM 2. If $n \geq 5$, then the problem (12) in the ball $B_{1}$ with the initial condition $Q_{0}(\cdot)$ satisfying the Hölder condition with exponent $1-2 / n$ 
on $[0,1]$, together with $\frac{d}{d y} Q_{0}(1)<\infty$ and the smallness condition $Q_{0}(y) \leq$ $((n-4) /(n-2)) 2 \sigma_{n} y^{1-2 / n}, y \in[0,1]$, has a classical solution global in time. Moreover, $Q(\cdot, t)$ converges to a stationary solution as $t \rightarrow \infty$, uniformly in $y$.

Proof. The strategy of the proof is the following: we construct a solution $Q=Q_{\varepsilon}$ to a regularized uniformly parabolic problem in $[0,1] \times[0, T]$, $T>0$ arbitrary, then we pass to the limit $\varepsilon \rightarrow 0$ on $[\delta, 1] \times[0, T]$, for any $\delta>0$, obtaining a classical solution of (12) global in time.

Note that the assumption on $Q_{0}(y)$ is a low concentration condition in the sense of [5, Section 2], which, however, allows us to consider some singular densities, e.g. $u_{0}(r) \leq 2(n-4) r^{-2}$.

We begin with a construction of supersolutions and subsolutions of the regularized uniformly parabolic problem

$$
\left(\mathcal{P}_{\varepsilon}\right) \quad Q_{t}=n^{2}(y+\varepsilon)^{2-2 / n} Q_{y y}+n \sigma_{n}^{-1} Q Q_{y}, \quad \varepsilon>0
$$

with the same boundary conditions as for (12) and smooth initial conditions $Q_{\varepsilon}(y, 0)$ converging to the one in $(12)$ in $C^{1-2 / n}([0,1])$.

Let $\bar{Q}_{\varepsilon}(y)=2 \sigma_{n}(y+\varepsilon)^{1-2 / n}, \varepsilon \geq 0$. The function $\bar{Q}_{\varepsilon}$ solves (15), while $\bar{Q}_{0}(y)=2 \sigma_{n} y^{1-2 / n}$ is a stationary solution of (12) corresponding to the singular solution $U(r)=2(n-2) r^{-2}$. It is standard to verify that for $\beta \in(0,1]$,

$$
Q_{\varepsilon, \beta}(y)=\min \left(\beta \bar{Q}_{\varepsilon}(y), M\right)
$$

is a supersolution to (15) with suitably small initial and boundary data in (12): $Q_{\varepsilon}(y, 0) \leq Q_{\varepsilon, \beta}(y)$. It is also easy to verify that (12) and (15) have nontrivial subsolutions of the form $\max \left(0, \gamma\left(y^{\kappa}-1\right)+M\right)$ with $\kappa \in(0,1)$ and suitably small $\gamma$. Here the assumption $\frac{d}{d y} Q_{0}(1)<\infty$ is used.

We note that standard arguments (cf. [9]) show that the principle of comparison with the above super- and subsolutions is valid for solutions of the regularized problem (15) (the key property is the boundedness of the derivatives of these super- and subsolutions).

For any solution $Q$ of $(15)$ define $P(y, t)=\frac{d}{d y} Q(y, t)$. The function $P$ satisfies the uniformly parabolic equation

(17) $P_{t}=n^{2}(y+\varepsilon)^{2-2 / n} P_{y y}+2 n(n-1)(y+\varepsilon)^{1-2 / n} P_{y}+n \sigma_{n}^{-1}\left(Q P_{y}+P^{2}\right)$ with $P(0, t) \geq 0, P(1, t) \geq 0$, and $P(y, 0) \geq 0$ (since the initial conditions for (12) and (15) are nondecreasing functions). Now the crucial observation is that $P_{\varepsilon, \beta}=\frac{d}{d y} Q_{\varepsilon, \beta}$ is a supersolution to (17) if $\beta \in(0,(n-4) /(n-2)]$. For the computation showing this we neglect the term $Q \frac{d}{d y} P_{\varepsilon, \beta} \leq 0$, and since in general no bound for $Q$ from below is available, the above smallness condition for $\beta$ appears.

The considerations above, together with [14, Ch. VI, Theorem 5.2], imply the existence of a unique solution of the problem $\left(\mathcal{P}_{\varepsilon}\right), Q=Q_{\varepsilon} \in$ 
$C^{2+\alpha, 1+\alpha / 2}([0,1] \times[0, T])$, for each $\alpha \in(0,1)$ and each $T>0$. This solution satisfies the estimates

$$
0 \leq Q_{\varepsilon}(y, t) \leq Q_{\varepsilon, \beta}(y), \quad 0 \leq \frac{d}{d y} Q_{\varepsilon}(y, t) \leq \frac{d}{d y} Q_{\varepsilon, \beta}(y),
$$

and for each $\delta>0$,

$$
\left\|Q_{\varepsilon}\right\|_{C^{1-2 / n, 1 / 2-1 / n}([\delta, 1] \times[0, T])} \leq C(\delta)
$$

with $C(\delta)$ independent of $\varepsilon$.

The compactness of the family of approximating solutions $\left\{Q_{\varepsilon}: \varepsilon>0\right\}$ is a consequence of their uniform continuity on $[0,1] \times[0, T]$. The existence of a common modulus of continuity follows from (18) applied for small $y$ and combined with (19) for $y \geq \delta$. By the above result, there is a sequence $\varepsilon_{k} \rightarrow 0$ and a function $Q \in C([0,1] \times[0, T])$ such that $Q_{\varepsilon_{k}}(y, t) \rightarrow Q(y, t)$ uniformly as $\varepsilon_{k} \rightarrow 0$ and $Q$ satisfies the initial and boundary conditions in (12).

Next let $(y, t) \in(0,1) \times(0, T)$. It follows from standard results ([14, Ch. III, Theorem 10.1] and [7, Ch. 3]) that $\left\{Q_{\varepsilon}: \varepsilon>0\right\}$ is bounded in the space of Hölder continuous functions $C^{m+\alpha,(m+\alpha) / 2}([y / 2,1] \times[t / 2, T])$ for each integer $m \geq 0$ and $\alpha \in(0,1)$. Then, since $Q_{\varepsilon}$ satisfies $(15)$ in $[y / 2,1] \times$ $[t / 2, T]$, it follows that $Q$ satisfies $(12)$ in $[y / 2,1] \times[t / 2, T]$. Finally, since $(y, t)$ was chosen arbitrarily in $(0,1) \times(0, T), Q$ satisfies $(12)$ in $(0,1) \times(0, T)$.

As a consequence of (18) the limit solution $Q$ and its derivative $\frac{d}{d y} Q$ can be estimated from above:

$$
\begin{aligned}
Q(y, t) & \leq \beta \bar{Q}_{0}(y)=\beta 2 \sigma_{n} y^{1-2 / n}, \\
\frac{d}{d y} Q(y, t) & \leq \beta \frac{d}{d y} \bar{Q}_{0}(y)=\beta 2 \sigma_{n}(1-2 / n) y^{-2 / n} .
\end{aligned}
$$

These upper bounds in (20) permit us to define an analogue of the Lyapunov function studied in $[5,(23)]$ :

$$
W=\int_{0}^{1} Q_{y} \log Q_{y} d y-\left(2 n \sigma_{n}\right)^{-1} \int_{0}^{1} y^{2 / n-2} Q^{2} d y .
$$

The same bounds (20) show that $W$ is uniformly bounded on the solutions constructed above. Moreover, $\frac{d}{d t} W(t) \leq 0$, and $\frac{d}{d t} W(t)=0$ if and only if $Q$ is a stationary solution (cf. [5, Section 2]). Finally, if $M<2 \sigma_{n}$ (here we have assumed $\left.M \leq((n-4) /(n-2)) 2 \sigma_{n}\right)$, by Theorem 1 stationary solutions are isolated, hence $Q(\cdot, t)$ converges as $t \rightarrow \infty$ to one of them.

Note that the sequence of approximating solutions can also be constructed for $M \leq 2 \sigma_{n}$, i.e. with $\beta=1$. In this case, however, we cannot prove any estimate for the derivative of the limit solution. In particular, we do not know whether the Lyapunov function is defined for a limit function $Q$, so the asymptotic behavior of such a solution is difficult to control. 
Remark. The arguments at the beginning of the proof of Theorem 2 (and references to [7], [14]) produce a unique classical Hölder continuous solution to a counterpart of (6)-(7) in an annulus, or in a spherical shell $\left\{x \in \mathbb{R}^{n}: 0<a<|x|<R\right\}, n \geq 2$, with Hölder continuous initial data of arbitrary size. The convergence to the stationary states (which are unique in the class of radial functions, cf. Section 2) follows exactly as at the end of the proof of Theorem 2 above.

4. Blowing-up solutions. The main result in this section shows that the smallness assumption in Theorem 2 is essential: there are local radial solutions of the evolution system in the ball which cannot be continued to global ones. Below we consider either the system (1)-(4) in the ball $B_{R} \subset \mathbb{R}^{n}, n \geq 2$, with a radially symmetric initial condition (5) and its solution constructed in [5, Theorem 2(i), (ii)], or the equation (12) and its classical solution whenever it exists. A sufficient condition for blow-up is more precise than that obtained in $[5$, Theorem $2(\mathrm{v})]$ for general star-shaped domains. The idea of the proof is close to that in the general case.

THEOREM 3. If the initial condition has sufficiently large concentration so that $M>2 n \sigma_{n} R^{n-2}, n \geq 2$, then the solution of the evolution system in the ball $B_{R} \subset \mathbb{R}^{n}$ ceases to exist after a finite time $T=T(M, R, n)$.

P r o of. With no loss of generality we may suppose $R=1$ as in the proofs of Theorems 1 and 2 . For $\alpha \in[2 / n-1,0]$ consider an auxiliary function

$$
w(t)=\int_{0}^{1} Q(y, t) y^{\alpha} d y .
$$

It can be easily checked that for any solution of (12),

$$
\begin{aligned}
\frac{d w}{d t}= & \left.n^{2} y^{2-2 / n+\alpha} Q_{y}\right|_{0} ^{1}-n^{2}(2-2 / n+\alpha) \int_{0}^{1} y^{1-2 / n+\alpha} Q_{y} d y \\
& +n \sigma_{n}^{-1} \int_{0}^{1} \frac{1}{2}\left(Q^{2}\right)_{y} y^{\alpha} d y .
\end{aligned}
$$

After another integration by parts, in the class of solutions considered, we obtain

$$
\begin{aligned}
\frac{d w}{d t} \geq & 0-\left.n(2 n-2+n \alpha) y^{1-2 / n+\alpha} Q\right|_{0} ^{1} \\
& +n(2 n-2+n \alpha)(1-2 / n+\alpha) \int_{0}^{1} y^{\alpha-2 / n} Q d y \\
& +\left.n\left(2 \sigma_{n}\right)^{-1} y^{\alpha} Q^{2}\right|_{0} ^{1}-\alpha n\left(2 \sigma_{n}\right)^{-1} \int_{0}^{1} y^{\alpha-1} Q^{2} d y
\end{aligned}
$$


In the admissible range of $\alpha$ 's this gives

$$
\begin{aligned}
\frac{d w}{d t} & \geq\left. n\left(2 \sigma_{n}\right)^{-1} y^{\alpha} Q\left(Q-2 \sigma_{n}(2 n-2+n \alpha) y^{1-2 / n}\right)\right|_{0} ^{1} \\
& =n\left(2 \sigma_{n}\right)^{-1} M\left(M-2 \sigma_{n}(2 n-2+n \alpha)\right),
\end{aligned}
$$

so in particular, for $\alpha=2 / n-1, M>2 n \sigma_{n}$ leads to unbounded growth of $w(t)$ in time. But this is absurd in view of a crude estimate $w(t) \leq$ $M \int_{0}^{1} y^{\alpha} d y=M(\alpha+1)^{-1}$. Remember that under the assumptions on $M$ of Theorem 3 stationary solutions do not exist.

Replacing $\alpha=2 / n-1$ by a larger $\alpha \leq 0$ we can obtain an analogous nonexistence result for less regular solutions of (1)-(5) or (12), but under a stronger condition $M>2 \sigma_{n}(2 n-2+n \alpha)$. In particular, taking $\alpha=0$ we only need $u(t) \in L^{1}\left(B_{R}\right), \lim _{|x| \rightarrow 0}|x|^{2(n-1)} u(x, t)=0$, hence some singularities of $u$ are admitted.

Finally, we infer from the local regularity of solutions in [5, Theorem 2(ii)] that the density $u$ cannot be bounded at the blow-up time $T$. In fact, the local smoothing effect shows that $u$ blows up simultaneously in $L^{\infty}\left(B_{R}\right)$ and in $L^{p}\left(B_{R}\right)$ for every $p>n / 2$ (recall [5, Theorem 2]).

To end this section, we make some remarks on special singular solutions to the system (1)-(2) or (6) considered without boundary conditions. This should help to understand the behavior of blowing-up solutions in Theorem 3 .

It is instructive to study the stationary solutions neither in the formulation given by $[5,(11)]$ nor (8) but (9) (with a nondecreasing $Q(r) \geq 0$ instead of the boundary condition), since the latter admits also some singular solutions $U, \Phi$.

The obvious solution $Q(r)=c=$ const $\geq 0$ corresponds for all $n \geq 2$ to a very singular $U=c \delta_{0}$. For $n=2$ we can also produce a family of other solutions $Q \geq 0$ of (9) with the boundary condition $Q(1)=Q_{0}$, which are determined by measures $u$. The construction follows in a particularly simple way from the observation that (9), by using a new independent variable $y=r^{2}$, transforms into $4 \pi y Q_{y y}+Q Q_{y}=0$. This equation (the stationary version of (12) for $n=2)$ is easily integrable:

$$
8 \pi y Q_{y}+Q(Q-8 \pi)=2 \pi c_{0} \quad \text { for some constant } c_{0} .
$$

Then, if $2 \pi c_{0}+8 \pi Q-Q^{2}=\left(Q-Q_{1}\right)\left(Q_{2}-Q\right)$, i.e. when $Q_{1,2}=\pi(4 \mp(16+$ $\left.\left.2 c_{0} / \pi\right)^{1 / 2}\right)$ for $c_{0} \in(-8 \pi, 0)$, another integration gives $\left(Q-Q_{1}\right) /\left(Q_{2}-Q\right)=$ $c y^{\beta}$ with $\beta=\left(1+c_{0} /(8 \pi)\right)^{1 / 2} \in(0,1)$. The explicit formula for $Q$ is

$$
\begin{gathered}
Q(y)=\left(Q_{1}+Q_{2} c y^{\beta}\right) /\left(1+c y^{\beta}\right), \\
0 \leq Q_{1}=Q(0) \leq Q(y) \leq Q(1)<Q_{2}<8 \pi
\end{gathered}
$$


so the mass concentrated at $y=0$ is $Q_{1} \in(0,4 \pi)$, and the total mass is $Q_{0} \in\left(Q_{1}, Q_{2}\right)$, and the latter, just as for radial regular solutions of [5, (11)] studied by means of (8), is strictly less than $8 \pi$ (cf. also [19, Lemma 4.5]).

For $n \geq 3$, besides these measure solutions, there are singular but integrable solutions $U(r)=2(n-2) r^{-2}$ corresponding to $Q(r)=2 \sigma_{n} r^{n-2}$. In the physically most relevant case $n=3$ this is the famous solution found by S. Chandrasekhar in the thirties when he studied the stellar structure. This is the unique radial solution with a positive singularity at 0 , but there is a surprising result of [1] on existence and an elegant classification of a rich variety of nonradial singular solutions to $[5,(11)]$ for $n=3$. Note that the solutions with $u(r) \sim r^{-2}$ cannot be studied in the framework of $[5$, Theorem 1], since $u \in L^{p}(B)$ for $p<n / 2$ only.

The next class of special solutions, more complicated than the stationary ones, are the self-similar solutions of the Boltzmann form

$$
u(r, t)=(T-t)^{-1} \xi\left(r(T-t)^{-1 / 2}\right) .
$$

Passing to the integrated density $Q(r, t)=\sigma_{n} \int_{0}^{r} \varrho^{n-1} u(\varrho, t) d \varrho$ we obtain $Q(r, t)=\sigma_{n}(T-t)^{n / 2-1} \zeta\left(r(T-t)^{-1 / 2}\right)$ for some new function $\zeta$ satisfying

$$
\begin{aligned}
\zeta^{\prime \prime}-(s / 2+(n-1) / s) \zeta^{\prime}+(n / 2-1) \zeta & +s^{1-n} \zeta \zeta^{\prime}=0, \\
s & =r(T-t)^{-1 / 2}, \quad '=d / d s .
\end{aligned}
$$

The equation (23) has explicit solutions:

$$
\begin{array}{lll}
\text { for } n=2: & \zeta(s)=\text { const } \geq 0 ; & \zeta(s)=s^{2} / 2 ; \\
\text { for } n \geq 3: & \zeta(s)=2 s^{n-2} ; & \zeta(s)=s^{n} / n .
\end{array}
$$

Except for the first one they satisfy the condition $\zeta(0)=0$, which means that no mass concentrates at the origin. These solutions correspond to

$$
\begin{aligned}
& (n=2) u=c \delta_{0} ; Q(r, t)=2 \pi r^{2} /(2(T-t)) \text { so } u(r, t)=1 /(T-t) ; \\
& (n \geq 3) u(r, t)=2(n-2) r^{-2} ; \\
& Q(r, t)=\sigma_{n} r^{n} /(n(T-t)) \text { so } u(r, t)=1 /(T-t),
\end{aligned}
$$

respectively. A natural question which may be posed in connection with self-similar solutions is: Are they stable among other blowing-up solutions?

The precise meaning of the stability of these (unbounded) objects can be given using the considerations from [8] for semilinear heat equations and from [2], [3] for the porous medium equation. Namely, after rescaling the time variable to $\tau=-\log (T-t)$ and putting $Q(r, t)=Q\left(s(T-t)^{1 / 2}, t\right)=$ $\sigma_{n}(T-t)^{n / 2-1} Z(s, \tau)$, where $s=r(T-t)^{-1 / 2}$ as before, (6) becomes

$$
Z_{\tau}=Z_{s s}-(s / 2+(n-1) / s) Z_{s}+(n / 2-1) Z+s^{1-n} Z Z_{s} .
$$

Now, the (local) stability of $\zeta$ solving (23) is interpreted as the stability in a suitable function space of $\zeta$ as a stationary solution of the parabolic 
equation (25) in $(s, \tau) \in \mathbb{R}^{+} \times \mathbb{R}^{+}$as $\tau \rightarrow \infty$. We will not pursue the above nontrivial question here, because the self-similar solutions (23) are of limited physical interest, since they do not satisfy the boundary conditions in (7). Indeed, it can be proved that for $\zeta$ satisfying (23) and $0 \leq \zeta \not \equiv$ const we have $\lim _{s \rightarrow \infty} \zeta(s)=\infty$. Their importance is connected rather with the local behavior near $r=0$ and $t \rightarrow T^{-}$.

Acknowledgements. The authors would like to thank Michiel Bertsch, Jan Goncerzewicz, Waldemar Hebisch, Andrzej Krzywicki and Michel Pierre for interesting discussions. We also thank PawełDaszkiewicz and Hiroshi Matano for helpful remarks.

\section{REFERENCES}

[1] M.-F. Bidaut-Véron and L. Véron, Nonlinear elliptic equations on compact Riemannian manifolds and asymptotics of Emden equations, Invent. Math. 106 (1991), 489-539.

[2] P. Biler, Blowup in nonlinear parabolic equations, Colloq. Math. 58 (1989), 85-110.

[3] - Blow-up in the porous medium equation, in: Recent Advances in Nonlinear Elliptic and Parabolic Problems, Proc. Internat. Conf. Nancy, 1988, P. Bénilan, M. Chipot, L. C. Evans and M. Pierre (eds.), Pitman Res. Notes in Math. 208, Longman, Harlow, 1989, 28-38.

[4] - Existence and asymptotics of solutions for a parabolic-elliptic system with nonlinear no-flux boundary conditions, Nonlinear Anal. 19 (1992), 1121-1136.

[5] P. Biler and T. Nadzieja, Existence and nonexistence of solutions for a model of gravitational interaction of particles, I, Colloq. Math. 66 (1994), 319-334.

[6] E. Caglioti, P.-L. Lions, C. Marchioro and M. Pulvirenti, A special class of stationary flows for two-dimensional Euler equations: a statistical mechanics description, Comm. Math. Phys. 143 (1992), 501-525.

[7] A. Friedman, Partial Differential Equations of Parabolic Type, Prentice-Hall, Englewood Cliffs, N.J., 1964

[8] Y. Giga and R. V. Kohn, Asymptotically self-similar blow-up of semilinear heat equations, Comm. Pure Appl. Math. 38 (1985), 297-319.

[9] D. Hilhorst, A nonlinear evolution problem arising in the physics of ionized gases, SIAM J. Math. Anal. 13 (1982), 16-39.

[10] D. D. Joseph and T. S. Lundgren, Quasilinear Dirichlet problems driven by positive sources, Arch. Rational Mech. Anal. 49 (1973), 241-269.

[11] A. Krzywicki and T. Nadzieja, Some results concerning the Poisson-Boltzmann equation, Zastos. Mat. 21 (1991), 265-272.

[12] - - - A nonstationary problem in the theory of electrolytes, Quart. Appl. Math. 50 (1992), 105-107.

[13] _, - A note on the Poisson-Boltzmann equation, Zastos. Mat. 21 (1993), 591-595.

[14] O. A. Ladyženskaja, V. A. Solonnikov and N. N. Ural'ceva, Linear and Quasilinear Equations of Parabolic Type, Amer. Math. Soc., Providence, R.I., 1988.

[15] K. Nagasaki and T. Suzuki, Radial and nonradial solutions for the nonlinear eigenvalue problem $\Delta u+\lambda e^{u}=0$ on annuli in $\mathbb{R}^{2}$, J. Differential Equations 87 (1990), 144-168. 
[16] K. Nagasaki and T. Suzuki, Radial solutions for $\Delta u+\lambda e^{u}=0$ on annuli in higher dimensions, ibid. 100 (1992), 137-161.

[17] F. Pacard, Radial and nonradial solutions of $-\Delta u=\lambda f(u)$ on an annulus of $\mathbb{R}^{n}$, $n \geq 3$, ibid. 101 (1993), 103-138.

[18] T. Suzuki, Global analysis for a two-dimensional elliptic eigenvalue problem with the exponential nonlinearity, Ann. Inst. Henri Poincaré Anal. Non Linéaire 9 (1992), 367-398.

[19] G. Wolansky, On steady distributions of self-attracting clusters under friction and fluctuations, Arch. Rational Mech. Anal. 119 (1992), 355-391.

[20] - On the evolution of self-interacting clusters and applications to semilinear equations with exponential nonlinearity, J. Analyse Math. 59 (1992), 251-272.

MATHEMATICAL INSTITUTE UNIVERSITY OF WROCŁAW PL. GRUNWALDZKI $2 / 4$ 50-384 WROCŁAW, POLAND
LABORATOIRE D'ANALYSE NUMÉRIQUE D'ORSAY UNIVERSITÉ DE PARIS-SUD BÂT. 425 91405 ORSAY, FRANCE

Reçu par la Rédaction le 31.1.1994 